\title{
EARTHQUAKE RESPONSE OF HIGH VOLTAGE CIRCUIT BREAKERS WITH NON-LINEAR FORCE-DEFLECTION CHARACTERISTICS
}

\author{
R. L. Mayes* and H. C. Hitchcock**
}

\section{Synopsis}

The New Zealand Electricity Department has purchased a number of high voltage circuit breakers with prestressed porcelain columns on which the manufacturers have carried out shock and vibration tests to determine strength and damping characteristics and to make some estimate of earthquake resistance. This paper presents a detailed study of the behaviour of such circuit breakers when subjected to earthquakes and steady-state sinusoidal forcing functions. The system, assumed to be single. degree of freedom, has a bilinear forcedeflection relationship. The system parameters that varied in this study were: natural period, damping, yield strength and elastic characteristics. All investigations were conducted with the aid of an IBM $360 / 40$ and an IBM 1130 digital computer using Jenning's Pseudo-Earthquakes $(1)$.

\section{Introduction}

The New Zealand Electricity Department has in recent years specified that the high voltage circuit breakers it purchases shall have a breaking strength at least twice that necessary to withstand a horizontal seismic load of 1.5 times its weight or some lesser value may be accepted if evidence is submitted as to the amount of equivalent viscous damping inherent in the equipment. The requirements are pased on a set of standard response spectra $(22)$ and are quite satisfactory for many items of equipment but become rather indefinite for structures with non-linear characteristics。

The Department has purchased a number of high voltage circuit breakers which have a high earthquake strength because their porcelain columns are prestressed by an inner insulating tension member as indicated in Fig. 1。 The makers had subjected the breaker to shock tests which induced accelerations up to $1.6 \mathrm{~g}$ at the top of the breaker and this was judged to provide a satisfactory degree of earthquake resistance.

However, with this type of construction circuit breakers have distinctly non-linear force-deflection characteristics with very low damping effect at large deflections. The Ph.D work of the first author has provided this opportunity of examining in greater detail the earthquake response of this unusual type of structure.

\section{Structure and Structural Parameters}

The structure under consideration was the

* Post Graduate Student, Auckland University.

* Senior Research Engineer, New Zealand Electricity Department, Wellington. high voltage circuit breaker shown in Fig. 1 which in service is mounted on a very rigid base. For the purpose of this study it was assumed that the ground motion is passed through this rigid base without modification, allowing the circuit breaker to be analysed as a single degree of freedom oscillatior.

Force deflection tests define the bilineal characteristics which arise when the bending overcomes the pretension and the porcelain column rocks on its heel. Forced vibration tests Fig. 2 and 3 show, for the $330 \mathrm{KV}$ version of the circuit breaker, the resonant frequencies, dynamic amplification and equivalent damping. They also show the shift in resonant frequency with amplitudes that exceed the "yield" point and the manufacturers have pointed out that "resonance" beyond yield point can only be achieved by controliing the displacement response and varying the input amplitude to suit.

The graphs indicate that damping is small at low amplitudes, increases briefly to about $5 \%$ with amplitudes just below "yield" point and becomes very small again with amplitudes above "yield" point. This is a long way from "viscous" damping behaviour and it is believed by the authors that for the purposes of this study the damping is best represented by a "Coulomb Damping Force" operative only up to "yield" displacement and of a size which gives the same energy dissipation per cycle as $2 \%$ viscous damping with an amplitude of vibration reaching yield point.

The preceding analysis of test results provides approximation of the force-deflection and damping characteristics of the circuit breaker. These factors were used for the more detailed earthquake analysis. The resultant force-deflection curves for this type of circuit breaker are shown in Fig. 4 .

Symbols used in the paper are listed in Table I.

Using the vibration test results together with information provided by the manufacturers on the masses of various components the values of these parameters were calculated for the 330 $\mathrm{kV}$ version of the circuit breaker and were deduced for the $220 \mathrm{kV}$ version. In each case values were obtained for the circuit breaker with and without the resistors (Item 2 Fig 1) which are not required for certain applications. The values of the key parameters are given in Tab1e II.

Because of limited computer time a full analysis was carried out only on the $220 \mathrm{KV}$ circuit breaker with resistors. 
302

\section{Steady - State Oscillation}

The dynamic response of structures to steady-state sinusoidal excitation can be obtained experimentally. Thus a study of the response amplitude as a function of frequency and maximum amplitude of the forcing function would be useful in either determining or verifying the parameters of the structure。

The steady-state solution of a single degree of freedom system with coulomb damping has been studied for an elastic system (3). A single degree of freedom system with a bilinear force-deflection relation has been studjed by the method of slowly-varying parameters $(4)$. A single degree of freedom system with Rambergosgood force-deflection relation has been studied by both the energy method $(5)$, and by slowly varying parameters $(5,6)$. The energy method is limited in scope for it gives the response at resonance only as it is only at this condition that the phase angle between the excitation and response is $\frac{\pi}{2}$. The results of the slowly-varying parameter method are considered in this section. This approach gives the steady-state response for all values of $\frac{\omega}{p}$ $i . e$. forcing frequency to the undamped natural frequency, and can be used to plot amplitude against frequency curves.

The equation of motion for the circuit breaker represented as a single degree of freedom oscillator in Fig. 5 is -

$m \ddot{x}+Q(x) \pm F_{c}=F(t)=F_{0} \operatorname{Cos} \omega t$

where $Q(x)$ is the elastic force and is a function of displacement; $F_{c}$ is the Coulomb damping force and is also a function of displacement, and + sign holds for mass moving in the positive $x$ direction, $F_{0}$ is the force amplitude and $\omega$ is the frequency of the excitation.

Letting $p^{2}=\frac{Q y}{x_{y} m} \quad n=\frac{\omega}{p} \quad \tau=p t$

The equation becomes

$m p^{2} \frac{d^{2} x}{d \tau^{2}}+Q(x) \pm F_{c}=F_{0} \cos \eta \tau$

Expressed in dimensionless form the equation of motion becomes

$\frac{d^{2}}{d \tau^{2}}\left(\frac{x}{x_{y}}\right)+\frac{x_{y}}{Q_{y}} Q\left(\frac{X}{x_{y}}\right) \pm \frac{F c}{Q_{y}}=\frac{F o}{Q_{y}} \cos \eta \tau$

where

$\frac{x^{y}}{Q_{y}} Q\left(\frac{x}{x_{y}}\right)=\frac{Q(x)}{Q_{y}}$

Letting the solution for equation 3 be

$\frac{x}{x_{y}}=\frac{x_{0}}{x_{y}} \cos \theta$

where $\theta=(\eta \tau+\phi)$ and $\frac{x_{0}}{x_{y}}$ and phase angle $\phi$ are

slowly varying parameters of $\tau$.
Apply the method of slowly-varying parameters $(8)^{y}$ (Appendix 1), equations 3 and 4 result in

$\left(\frac{\omega}{p}\right)^{2}=C\left(\frac{x_{0}}{x_{y}}\right) \pm$

$\sqrt{\frac{\left(F_{0} / Q_{y}\right)^{2}}{\left(x_{0} / x_{y}\right)^{2}}-\left(\frac{4}{\pi}\left(\frac{x_{y}}{x_{0}}\right) \frac{F_{C l}}{Q_{y}}\left(\left(1-\frac{x_{0}}{x_{y}}\right) R-1\right)\right)^{2}}$

where

$$
\begin{aligned}
& C\left(\frac{x_{0}}{x_{y}}\right)=\frac{2}{\pi} \quad(1-\epsilon) \frac{x_{y}}{x_{0}} \sqrt{1-\left(\frac{x_{y}}{x_{0}}\right)}- \\
& (1-\epsilon) \cos ^{-1}\left(\frac{x_{y}}{x_{0}}\right)+\frac{\pi}{2}
\end{aligned}
$$

The accuracy of equation 5 depends how close the actual response resembles a sinusoid, which itself depends upon the nature and amount of damping present. This will be discussed in a later section.

\section{Response to Random Excitation}

The more important response of the circuit breaker is that to an earthquake excitation. There are three methods of solution of the resulting non-linear equation of motion. The most common is that by the Runge-Kutta method which has been used by several different investigators for various force-deflection relationships $(5,7,8,9,10)$

Another method developed by Clough and Wilson(12), for multi-degree of freedom systems and non-linear force-deflection relationships uses a step-by-step integration procedure. This procedure is one of the most powerful digital computer techniques available for calculating the response of lumped-mass systems to arbitrary dynamic loads.

A method most suited to single degree of freedom systems and several times faster in most cases than the Runge-Kutta process, is a step-by-step process where the displacement and velocity at the beginning of a small interval of time are used to find the displacement, velocity and acceleration at the end of the interval( 11$)$. This was the method chosen for the analysis of the circuit breakers, it assumes that the earthquake accelerogram obeys a linear relationship between time intervals, so that

$$
\begin{aligned}
& \Delta t_{i}=t_{i+1}-t_{i} \\
& \Delta a=a_{i+1}-a_{i} \\
& \Delta t=t-t_{i}
\end{aligned}
$$

The equation of motion for the single degree of freedom oscillator represented in Fig 5 is

$m(\ddot{x}+\ddot{y})+Q(x) \pm F_{C}=0$

where tve sign holds for mass moving in tve direction.

The step by step solution of the above equation 
is

$$
\begin{aligned}
& \text { for }|x| \leqslant\left|x_{y}\right| \quad:- \\
& x_{i+1}=A(1,1) \cdot x_{i}+A(1,2) \cdot \dot{x}_{i}+B(1,1) \cdot a_{i}+ \\
& B(1,2) \cdot a_{i+1}+C(1,1) \cdot\left( \pm_{c 1}\right) \\
& \dot{x}_{i+1}=A(2,1) \cdot x_{i}+A(2,2) \cdot \dot{x}_{i}+B(2,1) \cdot a_{i}+ \\
& B(2,2) a_{i+1}+C(2,1) \cdot\left(t_{c 1}\right) \\
& \ddot{x}_{i+1}=-\left(p^{2} x_{i+1} \pm f_{c l}\right) . \\
& \text { for }|x|>\left|x_{y}\right| \quad:- \\
& x_{i+1}=D(1,1) \cdot x_{i}+D(1,2) \cdot x_{i}+E(1,1) \cdot a_{i}+ \\
& E(1,2) \cdot a_{i+1}+F(1,1) \cdot\left( \pm f_{C 2} \pm a_{y}\right) \\
& \dot{x}_{i+1}=D(2,1) \cdot x_{i}+D(2,2) \cdot \dot{x}_{i}+E(2,1) \cdot a_{i}+ \\
& E(2,2) \cdot a_{i+1}+F(2,1) \cdot\left( \pm f_{c 2} \pm a_{y}\right) \\
& \ddot{x}_{i+1}=-\left(p^{2} \varepsilon x_{i+1} \pm a_{y} \pm f_{c 2}\right)
\end{aligned}
$$

The $+v e$ sign of $a_{y}$ is for $x>x_{y^{*}}-v e$ sign is for $x<-x_{y}$.

Matrices A, B, C, D, E \& F are functions of $p, \Delta t, \varepsilon$ and are derived in Appendix II。 The preceding equations were solved on an IBM $360 / 40$ digital computer. As the maximum values of the response were of primary concern, rather than their complete time history, the computer was instructed to record the maximum values while evaluating them at.025 sec. time intervals.

\section{Verification of steady-State Response}

Using a sinusoidal forcing function in the step-by-step equation we are able to obtain the response time history of the circuit breakers, and compare the results with those obtained by the method of slowly-varying parameters. By summing the square of the difference between the actual response and the theoretical sinusoid with the same amplitude over 1 cycle, we obtain the square of the difference (SQD), which indicates how close the time history response is to a perfect sinusoid.

From $T_{a} b 1 e$ III it is quite clear that the method of slowly-varying parameters is not a good method of finding $\mathrm{x}_{0}$ if there is no damp. $\frac{x_{0}}{x_{y}}$

ing in the bilinear region $(\mathrm{R}=0)$ 。 $\mathrm{By}$ examining the appropriate values of $S Q D$, it is obvious that the assumption made, that the response is approximately sinusoidal is not valid in this case. However, if the damping is uniform in all regions $(R=1)$ then the method of slowly varying parameters is very useful as it gives a close approximation of $\frac{x_{0}}{x_{y}}$ found by the step-by-step methud because in this case the assumption that the response is approximately sinsuoidal is valid as can be seen by examining the appropriate values of $S Q D$.

\section{Earthquake Accelerograms}

When single body response spectra are used to define a design earthquake, they proride:

(a) an averaging of the responses to a number of different earthquakes (natural or artiricial) of a size likely to occur with significant frequency.

(b) a smoothing of these averaged ralues to indicate the probable average responses to a very large variety of similar earthquakes.

In using the response spectra it is essential to remember the wide range of possible response values which are implied especially when dealing with brittle structures.

The same point arises when using earthquake acceleration records to estimate the response of special structures not amenable to analysis with standard response spectra.

The present study aims to use a number of earthquake records but at the time of writing has used only two - B1 and D1 of Jennings series (1)。

The B1 earthquake represents the shaking to be expected near an Mo? earthquake and has the same spectral intensity as El Centro 1940. The D1 earthquake represents the shaking to be expected in the epicentral area of a short shallow earthquake of $\mathrm{M}_{0} 4$ to $\mathrm{M}_{0} 4.5$ magnitude and has a maximum ground acceleration of $.5 \mathrm{~g}$ (similar to that recorded at Parkfield, Calif. in 1966).

\section{Examination of Results}

The two earthquakes were applied to the mathematical model representing the $220 \mathrm{kV}$ version of the circuit breaker and the effect of design variations was explored by varying the primary natural period and the damping. The results, presented in Figs 7 to 10 , indicate:-

(1) The response of this type of structure appears to be as much or perhaps more affected by changes in parameters and earthquakes than that of the single linear elastic body.

(2) In Figs 7 and 8, reducing the yield level (or increasing the earthquake size) generally gives an increase in response but with a tendency to a rather sudden increase when the yield level is taken lower than about. $6 \mathrm{~g}$.

(3) In Figs 9 and 10, reducing the damping force generally gives a siight increase in response - again with a tendency to a sudden increase at some low value of damping.

(4) There is a pronounced selective effect with each earthquake - B1 excites the .215 sec structure much more strongly than the .143 sec or the .286 sec whereas Di clearly gives the .143 sec. structure the greatest excitation. 
304

Conclusions

If the estimates of the parameters of the $220 \mathrm{kV}$ breaker (fitted with resistors) are reasonably correct, its $.85 \mathrm{~g}$ acceleration response to the B1 (E1 Centro sized) earthquake, its $1.05 \mathrm{~g}$ response to the D1 (nearby small) earthquake together with its $1.6 \mathrm{~g}$ tested strength suggest that has a considerable margin of strength to cover the wide variations of actual earthquakes from the El Centro sized earthquake on which Departments specification is based。

The correct representation of damping is clearly very important and further comparison of calculated and measured response to a known sinusoidal excitation is needed to clarify the nature of the energy dispersal mechanism.

Some variants of this type of construction with lower yield levels and different spring stiffnesses could be expected to experience considerably greater relative deflections in similar sized earthquakes. Effective earthquake resistance would therefore depend not only on being able to withstand an induced acceleration at about the $1.5 \mathrm{~g}$ level but also on being able to withstand a large number of excursions beyond yield without significant damage either to itself or to equipment connected physically to it.

\section{Acknowledgement}

The authors thank G.E.C. Switchgear Ltd. for permission to reproduce the vibration test results for the English Electric "S" Frame 330 $\mathrm{kV}$ circuit breaker.

\section{References}

1. Jennings, P. C., G. W. Housner, and No C. Tsai, "Simulated Earthquake Motions", Californian Institute of Technology. Engineering Research Laboratory. April, 1968.

2。 Hitchcock, Ho C., "Electrical Equipment and Earthquakes", New Zealand Engineering, 24 (1): 3-14 (January 1969).

3. den Hartog, J. P. "Forced Vibrations with Combined Coulomb and Viscous Friction", ASME, 1931.

4. Caughey, T. K. "Sinusoidal Excitation of a System with Bilinear Hysteresis", Journal of Applied Mechanics, Vol. 27, No. 4, (Dec. $1960)$, pp。649-652.

5. Jennings, P. C., "Response of Simple Yielding Structures to Earthquake Excitation", Ph.D. Thesis, California Institute of Technology. Pasadena, California, 1963.

6. Minorski, N., "Non-Linear Mechanics", J.W. Edwards, Ann Arbor Michigan, 1947.

7. Berg, C。V. and Da Deppo, D. A., "Dynamic Analysis of Elasto-Plastic Structures", ASCE, EM2, April 1960.

8. Thomardes, S. S. "Earthquake Response of Systems with Bilinear Hysteresis" ASCE ST 4 , Aug. 1964.

9. Kaldjan, M. J. and Fan, Wo S. "Earthquake Response of a Ramberg Osgood Structure", ASCE ST10 Oct. 1968

10. Berg, G. V. and Thomardes, S. S. "Energy Comsumption by Structures in Strong Motion Earthquakes" 2nd World Conference on Earthquake Engineering, 1960.

11. Nigam, N. Co and Jennings, P, C. "Digital Calculation of Response Spectra from StrongMotion Earthquake Records". California
Institute of Technology. Earthquake Engineering Research Laboratory, 1968.

12. Wilson E. L. and Clough R。 W., "Dynamic Response by the Step-by-step Matrix Analysis", Symposium on Use of Computers in Civil Engineering, Lisbon, Portugal, oct. 1962

\section{Appendix}

Equation 3 is

$\frac{d^{2}}{d \tau^{2}}\left(\frac{x}{x_{y}}\right)+\frac{x_{y}}{Q_{y}} Q\left(\frac{x}{x_{y}}\right) \pm \frac{F}{Q_{y}}=\frac{F}{Q_{y}} \cos \eta \tau$

Letting the solution of 3 be

$\frac{x}{x_{y}}=\frac{x_{o}}{x_{y}} \cos \theta$

where $\theta=\eta \tau+\phi$ and $\frac{x_{0}}{x_{y}}$ and phase angle $\phi$ are slowly varying parameters of $\tau$.

$\frac{x^{\prime}}{x_{y}}=-\frac{x_{0}}{x_{y}} \eta \sin \theta+\frac{x_{0}^{\prime}}{x_{y}} \cos \theta-\frac{x_{0}}{x_{y}} \phi \cdot \sin \theta$

As is done in Lagrange's method of variation of parameters the last two terms are set equal to zero.

$\frac{x_{0}^{\prime}}{x_{y}} \cos \theta-\frac{x_{0}}{x_{y}} \phi \cdot \sin \theta=0$

From equation $3 \mathrm{~A}$ and $4 \mathrm{~A}$

$\frac{x^{\prime \prime}}{x_{y}}=-\frac{x_{0}}{x_{y}} n^{2} \cos \theta-\frac{x_{0}{ }^{\prime}}{x_{y}} n \sin \theta-\frac{x_{0}}{x_{y}} \phi \cdot \cos \theta 5 A$

Substituting $2 \mathrm{~A}$ and $5 \mathrm{~A}$ into $1 \mathrm{~A}$

$-\frac{x_{0}}{x_{y}} n^{2} \cos \theta-\frac{x_{0}{ }^{\prime}}{x_{y}} \eta \sin \theta-\frac{x_{o}}{x_{y}} \eta \phi \cdot \cos \theta+$

$\frac{x_{y}}{Q_{y}} Q\left(\frac{x_{o}}{x_{y}} \cos \theta\right) \pm \frac{F}{Q_{y}}=\frac{F_{o}}{Q_{y}} \cos \eta \tau$

multiply $4 \mathrm{~A}$ by $-n \cos \theta$ and 6 by $\sin \theta$ and add.

$-\frac{x_{0}}{x_{y}} n^{2} \sin \theta \cos \theta-\frac{x_{0}^{\prime}}{x_{y}} \eta\left(\sin ^{2} \theta+\cos ^{2} \theta\right)+$

$\frac{x_{y}}{Q_{y}} Q\left(\frac{x_{0}}{x_{y}} \cos \theta\right) \cdot \sin \theta \pm \frac{F_{c}}{Q_{y}} \sin \theta=$

$\frac{F_{0}}{Q_{y}} \cos \eta \tau \sin \theta$

multiply $4 \mathrm{~A}$ by $\eta \sin \theta$ and $6 \mathrm{~A}$ by $\cos \theta$ and add

$-\frac{x_{0}}{x_{y}} \eta^{2} \cos ^{2} \theta-\frac{x_{0}}{x_{y}} \eta \phi \cdot\left(\sin ^{2} \theta+\cos ^{2} \theta\right)+$

$\frac{\mathrm{x}_{\mathrm{y}}}{\mathrm{Q}_{\mathrm{y}}} \mathrm{Q}\left(\frac{\mathrm{x}_{\mathrm{o}}}{\mathrm{x}_{\mathrm{y}}} \cos \theta\right) \cdot \cos \theta \pm \frac{F_{c}}{\mathrm{Q}_{\mathrm{y}}} \cos \theta=\frac{F_{0}}{Q_{y}} \cos \eta \tau \cos \theta$ 
As $\frac{x_{0}}{x_{y}}$ and $\phi$ are slowly varying parameters the average of equations 7 and 8 are satisfied instead of the equation itselfo Averaging over 1 cycle, the damping terms must only be averaged over the portion they actually act, equation $7 \mathrm{~A}$ and $8 \mathrm{~A}$ become

$-2 \pi \frac{x_{0} \cdot}{x_{y}}+\frac{1}{\pi} \int_{0}^{2 \pi} \frac{x_{y}}{Q_{y}} Q\left(\frac{x_{0}}{x_{y}} \cos \theta\right) \cdot \sin \theta \cdot d \theta-$

$\frac{4}{\pi} \frac{F c 1}{Q_{y}}\left(\frac{x_{y}}{x_{o}}-\left(\frac{x_{y}}{x_{o}}-1\right) R\right)=\frac{F_{0}}{Q_{y}} \sin \theta$

$-\frac{x_{0}}{x_{y}} \eta^{2}-2 \eta \phi^{\prime} \frac{x_{0}}{x_{y}}+$

$\frac{1}{\pi} \int_{0}^{2 \pi} \frac{x_{y}}{Q_{y}} Q\left(\frac{x_{0}}{x_{y}} \cos \theta\right) \cdot \cos \theta \cdot d \theta=\frac{F}{Q_{y}} \cos \theta \quad 10 A$

Define $S_{\left(x_{0}\right)}$ and $C_{\left(x_{0}\right)}$ as

$c_{\left(x_{0}\right)}=\frac{x_{y}}{x_{0}} \cdot \frac{1}{\pi} \int_{0}^{2 \pi} \frac{x_{y}}{Q_{y}} Q\left(\frac{x_{0}}{x_{y}} \cos \theta\right) \cdot \cos \theta \cdot d \theta \quad 11 A$

$S\left(x_{0}\right)=\frac{x_{y}}{x_{0}} \cdot \frac{1}{\pi} \int_{0}^{2 \pi} \frac{x_{y}}{Q_{y}} Q\left(\frac{x_{0}}{x_{y}} \cos \theta\right) \cdot \sin \theta \cdot d \theta \quad 12 A$

For steady-state response, time derivatives of $\mathrm{x}_{0}$ and $\phi$ are zero: Hence steady-state

equations, from $9 \mathrm{~A}$ and $10 \mathrm{~A}$ are

$-\frac{4}{\pi} \frac{F c l}{Q_{y}}\left(\frac{x_{y}}{x_{o}}-\left(\frac{x_{y}}{x_{o}}-1\right) R\right)+\frac{x_{o}}{x_{y}} s\left(x_{0}\right)=\frac{F}{Q_{y}} \sin \phi 13 A$

$-\frac{x_{o}}{x_{y}} n^{2}+\frac{x_{o}}{x_{y}} C\left(x_{o}\right)=\frac{F_{0}}{Q_{y}} \cos \phi$

Squaring both sides, and add.

$\left[S\left(x_{0}\right)-\frac{4}{\pi} \frac{F}{Q_{y}}\left(1-\left(1-\frac{x_{o}}{x_{y}}\right) R\right)\right]^{2}+\left[C\left(x_{0}\right)-n^{2}\right]^{2}=$

$\frac{\left(F_{0} / Q_{y}\right)^{2}}{\left(x_{0} / x_{y}\right)^{2}}$

$\eta^{2}=\left(\frac{\omega}{p}\right)^{2}=C\left(x_{0}\right) \pm$

$\sqrt{\frac{\left(F_{0} / Q_{y}\right)^{2}}{\left(x_{0} / x_{y}\right)^{2}}-\left[S\left(x_{0}\right)-\frac{4}{\pi} \frac{F_{C l}}{Q_{y}}\left(1-\left(1-\frac{x_{0}}{x_{y}}\right) R\right]^{2}\right.} 15 A$

$S\left(x_{0}\right)=\frac{x_{y}}{x_{0}} \cdot 2 \int_{0}^{\pi} \frac{x_{y}}{Q_{y}} Q\left(\frac{x_{0}}{x_{y}} \cos \theta\right) \cdot \sin \theta \cdot d \theta \underset{12 A}{\text { from }}$

$\frac{x_{0}}{x_{y}} \cos \theta=z$

from
$S\left(x_{0}\right)=-\left(\frac{x_{y}}{x_{0}}\right)^{2} \frac{2}{\pi}\left[\int_{\frac{x_{0}}{x_{y}}}^{1}((1-\epsilon)+\epsilon z) d z+\right.$

$\left.\int_{1}^{-1} z d z+\int_{-1}^{-\frac{x_{0}}{x_{y}}}(\epsilon z-(1-\epsilon)) d z\right]$

$\therefore \dot{s}\left(x_{0}\right)=0$.

$16 \mathrm{~A}$ $C\left(x_{0}\right)=\frac{x_{y}}{x_{0}} \cdot \frac{2}{\pi} \int_{0}^{\pi} \frac{x_{y}}{Q_{y}} Q\left(\frac{x_{0}}{x_{y}} \cos \theta\right) \cdot \cos \theta \cdot d \theta$ from With the same substitution as for $S\left(x_{0}\right)$

$C\left(x_{0}\right)=-\left(\frac{x_{y}}{x_{0}}\right)^{2} \frac{2}{\pi} \int_{\frac{x_{0}}{x_{y}}}^{1}((1-\epsilon)+\epsilon z) \sqrt{\frac{z d z}{\left(\frac{x_{0}}{x_{y}}\right)^{2}-z^{2}}}$

$+\int_{1}^{-1} z \frac{\frac{z d z}{2}}{\sqrt{\left(\frac{x_{0}}{x_{y}}\right)^{2}-z^{2}}}+\int_{-1}^{-\frac{x_{0}}{x_{y}}}(\epsilon z-(1-\epsilon)) \sqrt{\sqrt{\left(\frac{x_{0}}{x_{y}}\right)^{2}-z^{2}}}$

$C\left(x_{0}\right)=\frac{2}{\pi}\left[(1-\epsilon) \frac{x_{y}}{x_{0}} \sqrt{1-\left(\frac{x_{y}}{x_{0}}\right)^{2}}-(1-\epsilon) \cos ^{-1}\left(\frac{x_{y}}{x_{0}}\right)\right.$

$\left.+\frac{\pi}{2}\right]$

\section{APPENDIX II}

The resulting non-1inear equation for the single degree of freedom oscillator represented in Fig. 5 subjected to a random excitation is

$m(\ddot{x}+\ddot{y})+Q(x) \pm F_{C}=0$

$\ldots 1 \mathrm{~B}$

where + ve sign holds for the mass moving in the + ve $x$ direction.

$\ddot{y}=a_{i}+\frac{\Delta a_{i}}{\Delta t_{i}}\left(t-t_{i}\right)$

$\therefore \ddot{x}+q(x) \pm f_{c}=-a_{i}-\frac{\Delta a_{i}}{\Delta t_{i}}\left(t-t_{i}\right) \ldots 3 B$

where $q(x)$ and $f_{c}$ obey the following relationships。

$$
\begin{array}{ll}
|x| \leqslant x_{y} & x<-x_{y} \\
q(x)=p_{1}^{2} x & \left.q(x)=-a_{y}+p_{2}^{2} x\right) \\
f_{c}=f_{c l} & f_{c}=f_{c 2}=R f_{c 1}
\end{array}
$$


$x>x_{y}$

$q(x)=a_{y}+p_{2}^{2} x+f c$ holds for $\dot{x}+v 0$

$f_{c}=f_{c 2}=R_{0} f_{c l} \quad-f_{c}$ holds for $\dot{x}-$ ve

The solution of equation $3 \mathrm{~B}$ depends on the region of the elastic force $Q(x)$.

\section{A. Elastickegion $|x| \leqslant\left|x_{y}\right|$}

Equation of motion becomes

$\ddot{x}+p_{1}^{2} x \pm f_{c l}=-a_{i}-\frac{\Delta a_{i}}{\Delta t_{i}}\left(t-t_{i}\right)$

The full solution of this equation is :-

$x=A^{\cdot} \cos \left(p_{1}\left(t-t_{i}\right)\right)+B^{\cdot} \sin \left(p_{1}\left(t-t_{i}\right)\right)-$

$\frac{\left( \pm f_{c l}\right)}{p_{1}^{2}}-\frac{a_{i}}{p_{1}^{2}}-\frac{1}{p_{1}^{2}} \frac{\Delta a_{i}}{\Delta t_{i}}\left(t-t_{i}\right)$

$\dot{x}=-A^{\prime} p_{1} \sin \left(p_{1}\left(t-t_{i}\right)\right)+B^{\prime} p_{1} \operatorname{Cos}\left(p_{1}\left(t-t{ }_{i}\right)\right)-$

$\frac{1}{p_{1}^{2}} \frac{\Delta a_{i}}{\Delta t_{i}}$

at $t=t_{i} \quad x=x_{i}$ and $\quad \dot{x}=\dot{x}_{i}$

$\therefore \quad A^{\prime}=x_{i}+\left(\frac{ \pm f c_{1}}{p_{1}^{2}}+\frac{a_{i}}{p_{1}^{2}}\right.$

$$
B^{\prime}=\frac{\dot{x}_{i}}{p_{1}}+\frac{1}{p_{1}^{3}} \frac{\Delta a_{i}}{\Delta t_{i}}
$$

substituting $A^{\prime}$ and $B^{\prime}$ in $5 B$ and $6 B$ gives $x$ and $\dot{x}$ at $t=t_{i+1}$.

$x_{i+1}=A(1,1) \cdot x_{i}+A(1,2) \cdot \dot{x}_{i}+B(1,1) \cdot a_{i}+$

$B(1,2) \cdot a_{i+1}+C(1,1) \cdot\left( \pm f_{c 1}\right)$

$\dot{x}_{i+1}=A(2,1) \cdot x_{i}+A(2,2) \cdot \dot{x}_{i}+B(2,1) \cdot a_{i}+$

$B(2,2) \cdot a_{i+1}+C(2,1) \cdot\left( \pm f_{c 1}\right)$

$8 \mathrm{~B}$

where $\Delta t=t_{i+1}-t_{i}$

$A(1,1)=\operatorname{Cos}\left(p_{1} \Delta t\right)$

$A(1,2)=\frac{1}{p_{1}} \sin \left(p_{1} \Delta t\right)$

$A(2,1)=-p_{1} \operatorname{Sinp}_{1} \Delta t$

$B(1,1)=-\frac{1}{p_{1}^{3} \Delta t} \sin \left(p_{1} \Delta t\right)$

$B(1,2)=\frac{1}{p_{1}^{3} \Delta t} \sin \left(p_{1} \Delta t\right)$
$B(2,1)=\frac{1}{p_{1}^{2} \Delta t} \cos \left(p_{1} \Delta t\right)-\frac{1}{p_{1}} \sin \left(p_{1} \Delta t\right)+\frac{1}{p_{1}^{2} \Delta t}$

$B(2,2)=\frac{1}{p_{1}^{2} \Delta t} \cos \left(p_{1} \Delta t\right)-\frac{1}{p_{1}^{2} \Delta t}$

$C(1,1)=\frac{1}{p_{1}^{2}} \cos \left(p_{1} \Delta t\right)-\frac{1}{p_{1}^{2}} C(2,1)=$.

$\frac{1}{p_{1}} \sin \left(p_{1} \Delta t\right)$

B. Bilinear Region $|x|>\left|x_{y}\right|$

Equation of motion becomes

$\ddot{x}+p_{2}^{2} x \pm a_{y} \pm f_{c 2}=-a_{i}-\frac{\Delta a_{i}}{\Delta t_{i}}\left(t-t_{j}\right) \ldots 9 B$

where $+a_{y}$ holds for $x>x_{y}$

$+f_{c 2}$ holds when $\dot{x}$ is $+{ }_{*}$

$-a_{y}$ holds for $x<-x_{y}$.

- $f_{c 2}$ holds when $\dot{x}$ is - ve.

Full solution of this equation is

$x=A^{\prime \prime} \operatorname{Cos}\left(p_{d}\left(t-t_{i}\right)\right)+B^{\prime \prime} \operatorname{Sin}\left(p_{d}\left(t-t_{i}\right)\right)-\frac{a_{y}}{p_{1}^{2} \epsilon}-$

$\left( \pm \frac{f_{c}^{2}}{p_{1}^{2} \varepsilon}-\frac{a_{i}}{p_{1}^{2} \varepsilon}-\frac{1}{p_{1}^{2} \varepsilon} \cdot \frac{\Delta a_{i}}{\Delta t_{i}}\left(t-t_{i}\right) . \quad \ldots 10 B\right.$ $\dot{x}=-A^{n} p_{d} \operatorname{Sin}\left(p_{d}\left(t-t_{i}\right)\right)+B^{n} p_{d} \operatorname{Cos}\left(p_{d}\left(t-t_{i}\right)\right)-$

$\frac{1}{p_{1}^{2} \varepsilon} \frac{\Delta \mathrm{a}_{i}}{\Delta t_{i}}$

where $\varepsilon=\frac{\mathrm{k}_{2}}{\mathrm{k}_{1}}=\frac{\mathrm{p}_{2}^{2}}{\mathrm{p}_{1}^{2}}$ and $\mathrm{p}_{\mathrm{d}}=\mathrm{p}_{1} \sqrt{\varepsilon}$

at $t=t_{i} \quad x=x_{i} \quad$ and $\dot{x}=\dot{x}_{i}$

$\therefore \quad A^{\prime \prime}=x_{i}+\frac{a_{y}}{p_{d}^{2}}+\frac{( \pm f c 2)}{p_{d}^{2}}+\frac{a_{i}}{p_{d}^{2}}$

$B^{\prime \prime}=\frac{\dot{x}_{i}}{p_{d}}+\frac{1}{p_{d}^{3}} \frac{\Delta a_{i}}{\Delta t_{i}}$.

at $t=t_{i+1} \quad x=x_{i+1} \quad \dot{x}=\dot{x}_{i+1} \quad \Delta t=t_{i+1}-t$

$x_{i+1}=D(1,1) \cdot x_{i}+D(1,2) \cdot \dot{x}_{i}+E(1,1) \cdot a_{i}+$

$E(1,2) \cdot a_{i+1}+F(1,1) \cdot\left( \pm f c 2 \pm a_{y}\right)$

$\ldots 12 \mathrm{~B}$ 


$$
\begin{aligned}
& i_{i+1} \because D(2,1) \cdot \backslash_{i}+D(2,2) \cdot \dot{x}_{i}+F(2,1) \cdot a_{i}+ \\
& E(2,2) \cdot a_{i+1}+f(2,1) \cdot\left( \pm f c 2 \pm a_{y}\right) \quad \ldots 13 B \\
& \text { whers } D(1,1) \quad \cos \left(p_{d} \Delta t\right) \\
& D(2,1)=-D_{d} \sin \left(p_{d} \Delta t\right) \\
& D(1,2)=\frac{1}{p_{\mathrm{Cl}}} \sin \left(\mathrm{p}_{\mathrm{d}} \Delta \mathrm{t}\right) \\
& \left.D(2,2)=\cos (1)_{d} \Delta t\right) \\
& E(1,1)=\frac{1}{p d^{2}} \cos \left(p_{d} \Delta t\right)-\frac{1}{p^{3} \Delta t} \sin \left(p_{d} \Delta t\right) \\
& E(1,2)=-\frac{1}{p_{d}^{3} \Delta t} \sin \left(p_{d} \Delta t\right)-\frac{1}{p_{d}^{2}} \\
& E(2,1)=-\frac{1}{p_{d}} \sin \left(p_{d} \Delta t\right) \\
& E(2,2)=\frac{1}{p_{d}^{2} \Delta t} \operatorname{Cos}\left(p_{d} \Delta t\right)-\frac{1}{p_{d}^{2} \Delta t} \\
& F(1,1)=\frac{1}{p_{d}^{2}} \cos \left(p_{d} \Delta t\right)-\frac{1}{p_{d}^{2}} \\
& F(2,1)=-\frac{1}{p_{d}} \sin \left(p_{d} \Delta t\right) \text {. }
\end{aligned}
$$


TABLE I

LIST OF SYMBOLS

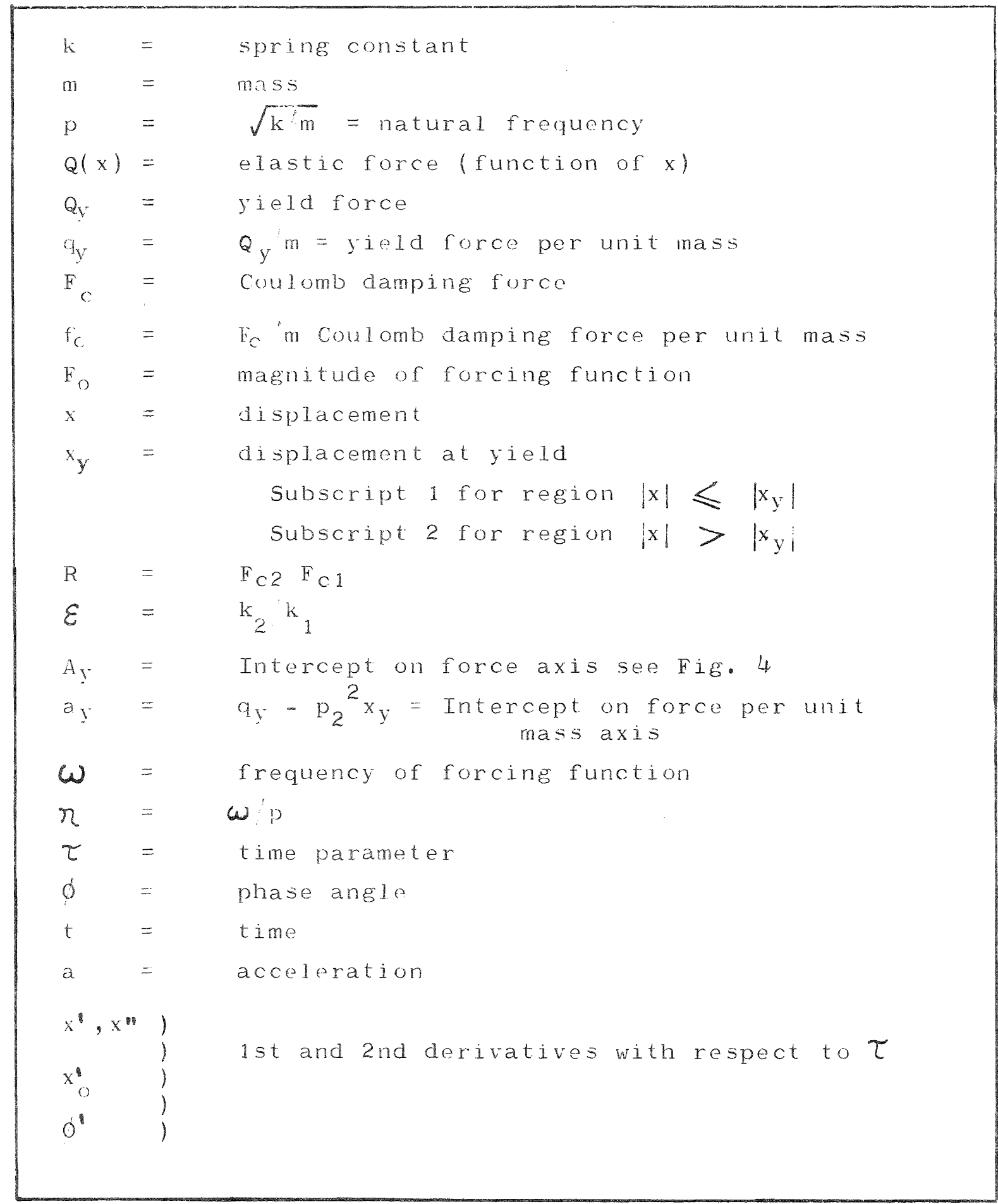


TAFSLE I I

\begin{tabular}{|c|c|c|c|c|}
\hline & \multicolumn{2}{|c|}{$330 \mathrm{kV}$} & \multicolumn{2}{|c|}{$220 \mathrm{kV}$} \\
\hline & $\begin{array}{c}\text { Wilh } \\
\text { Resistors }\end{array}$ & $\begin{array}{l}\text { Without } \\
\text { Resistor: }\end{array}$ & $\begin{array}{c}\text { With } \\
\text { Rosistors }\end{array}$ & $\begin{array}{l}\text { Without } \\
\text { Rosistors }\end{array}$ \\
\hline $\begin{array}{cl}\text { Primary } & \text { Natural } \\
\text { Periof (Sec) }\end{array}$ & .204 & .174 & $.14 \%$ & .116 \\
\hline $\begin{array}{c}\text { Equivalent Mass } \\
(\mathrm{kg})\end{array}$ & 807 & 587 & 831 & 557 \\
\hline $\begin{array}{l}\text { Yiold Force Unit } \\
\text { Mass (fraction } \\
\text { of gor }\end{array}$ & .72 & .99 & .90 & 1.35 \\
\hline $\begin{array}{l}\text { Stiffnoss ratjo } \\
=\mathrm{k} 2 / \mathrm{k} 1\end{array}$ & .128 & .128 & .10 & .10 \\
\hline $\begin{array}{l}\text { Coulomb Force! } \\
\text { Unil Mass } \\
\text { (fraction of g) }\end{array}$ & & & & \\
\hline$U_{p}$ lo Yjold $\left(f_{C 1} f_{\xi}\right)$ & .0226 & .031 & $.028 ?$ & .0422 \\
\hline Boyond Yiold f fra $g)$ & 0 & 0 & 0 & 0 \\
\hline
\end{tabular}


TABLE III

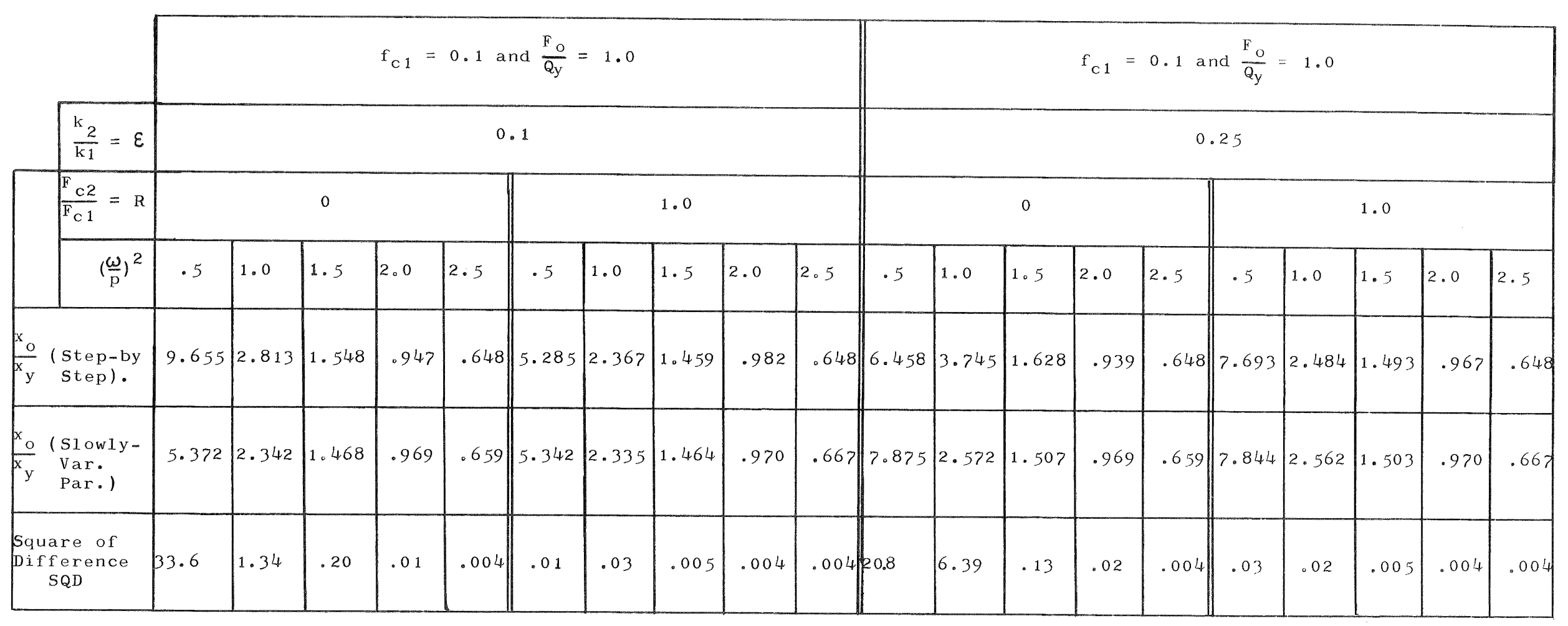



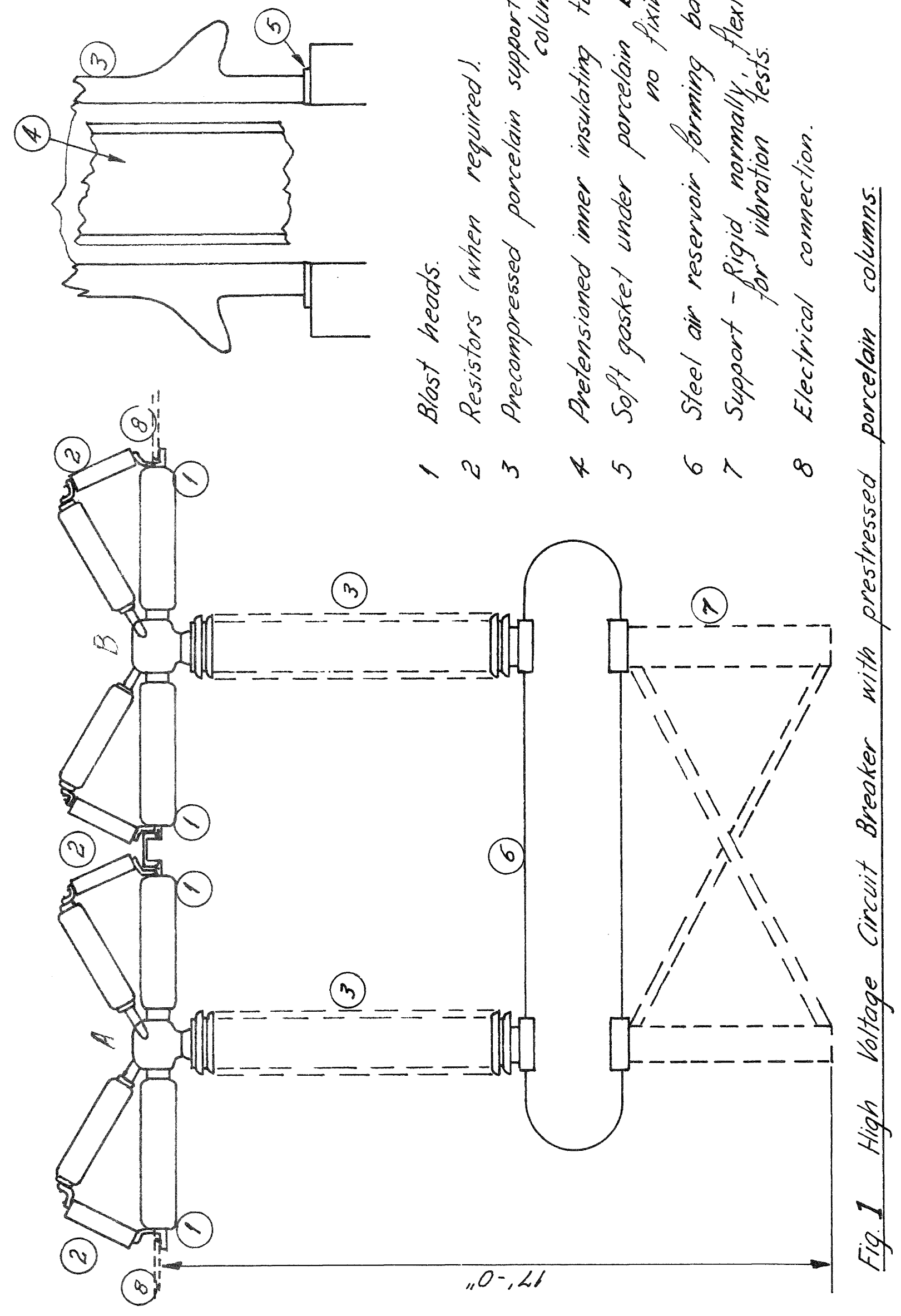
312

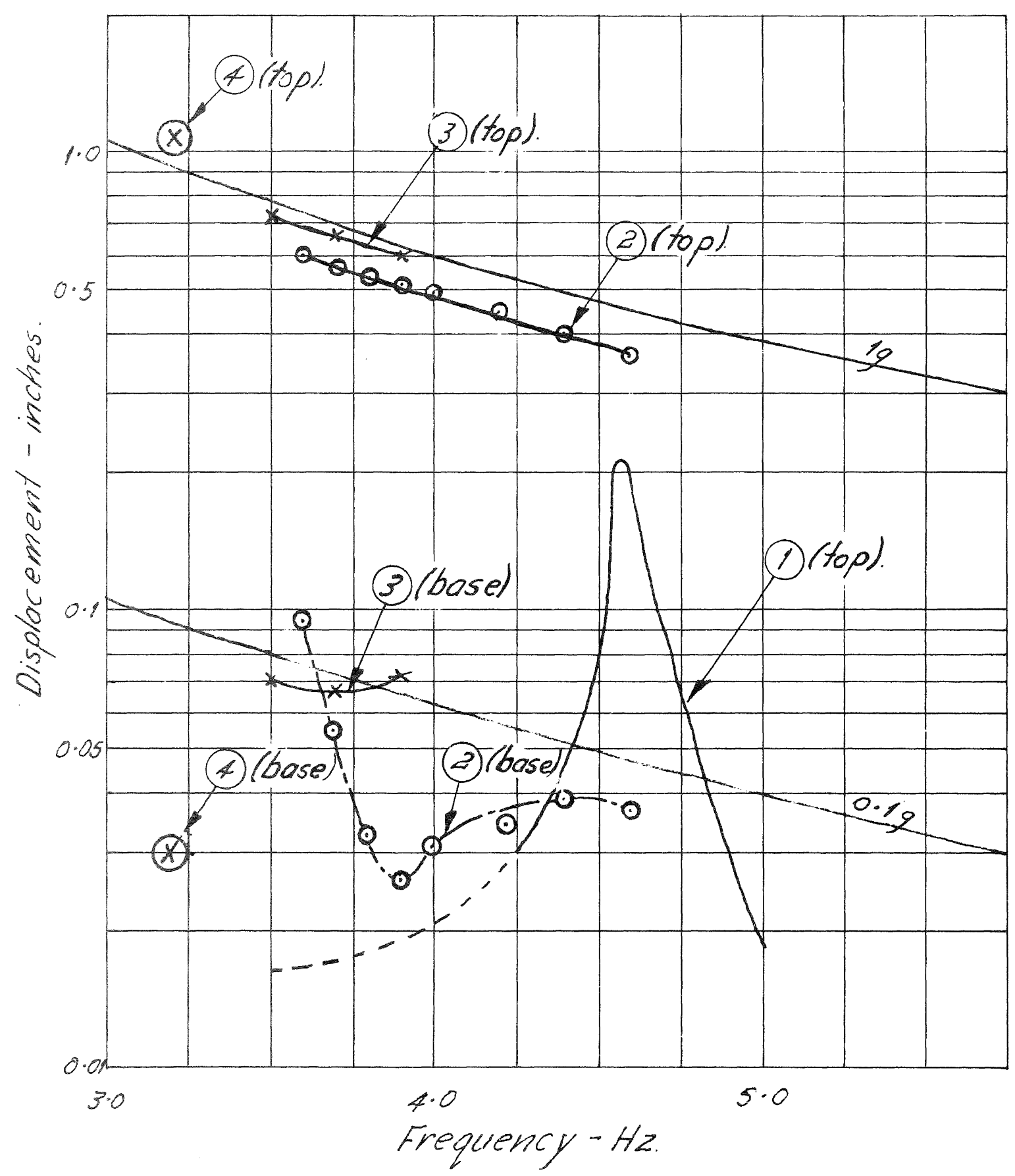

Fig. 2 Forced vibration tests on $330 \mathrm{kV}$ Circuit Breakers.

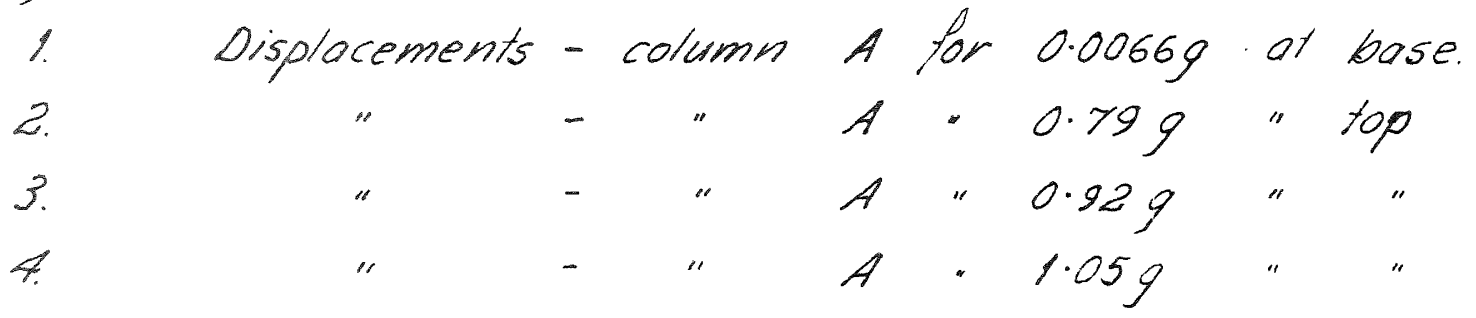




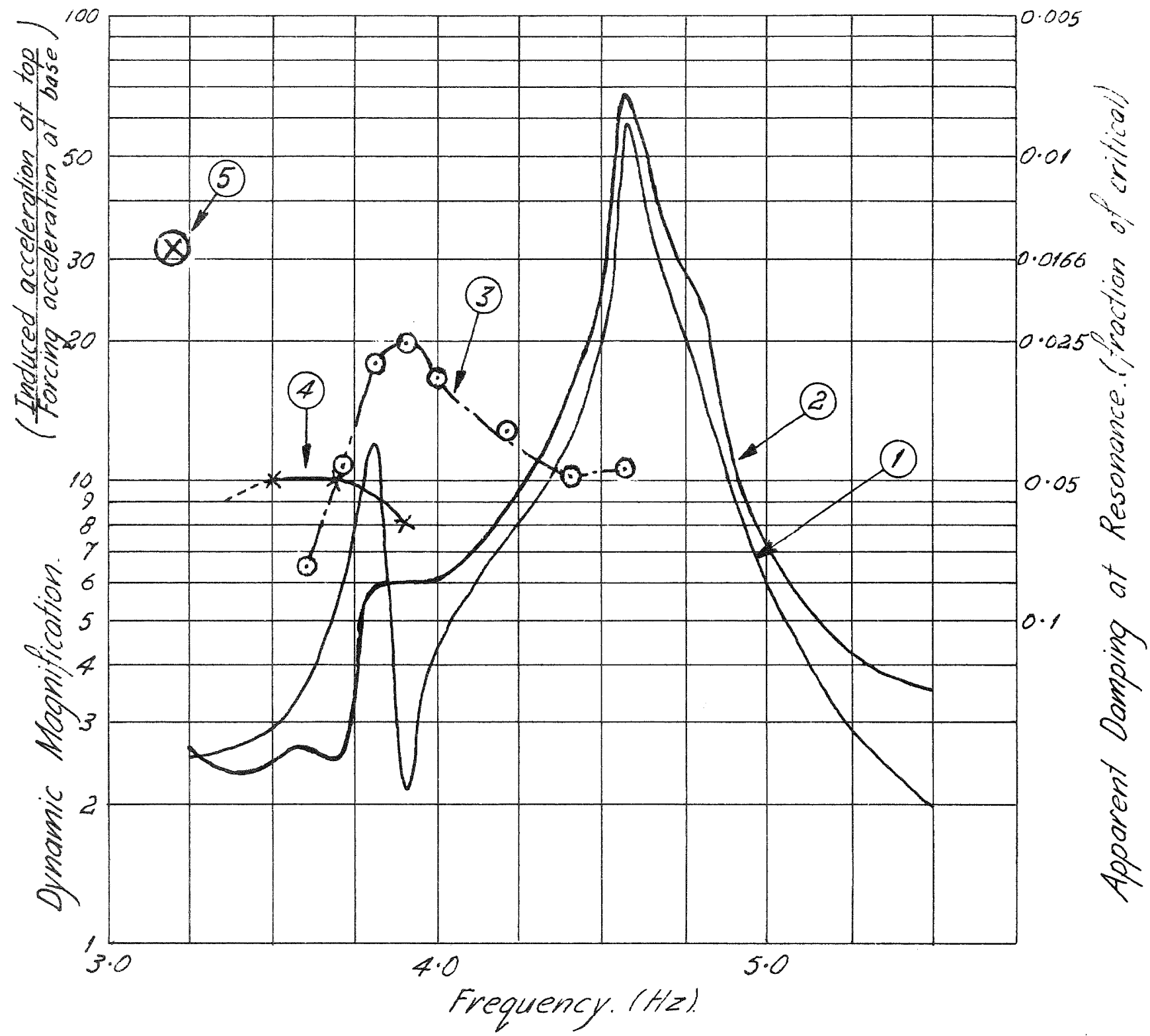

Fig. 3 Forced vibration tests on $330 \mathrm{kV}$ Circuit Breakers.

1. Oynamic Magnification - column A for $0.0066 \mathrm{~g}$ ot base.

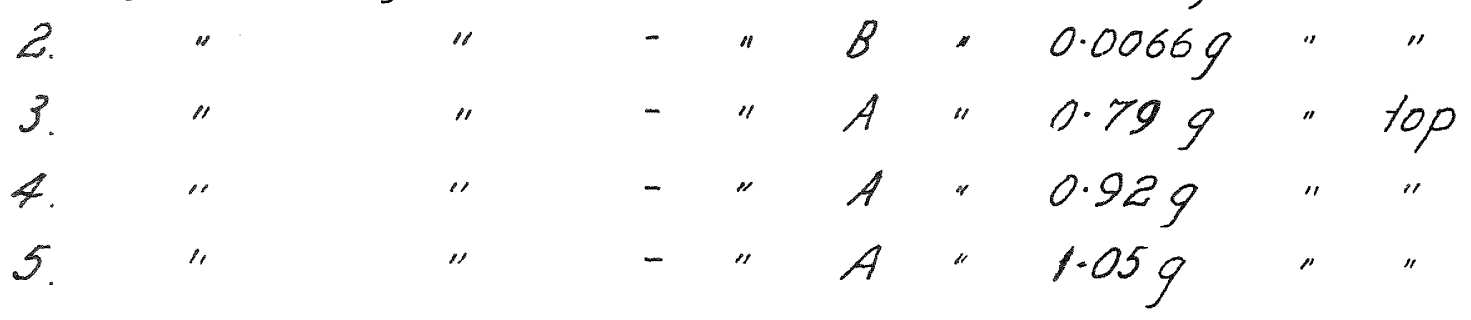



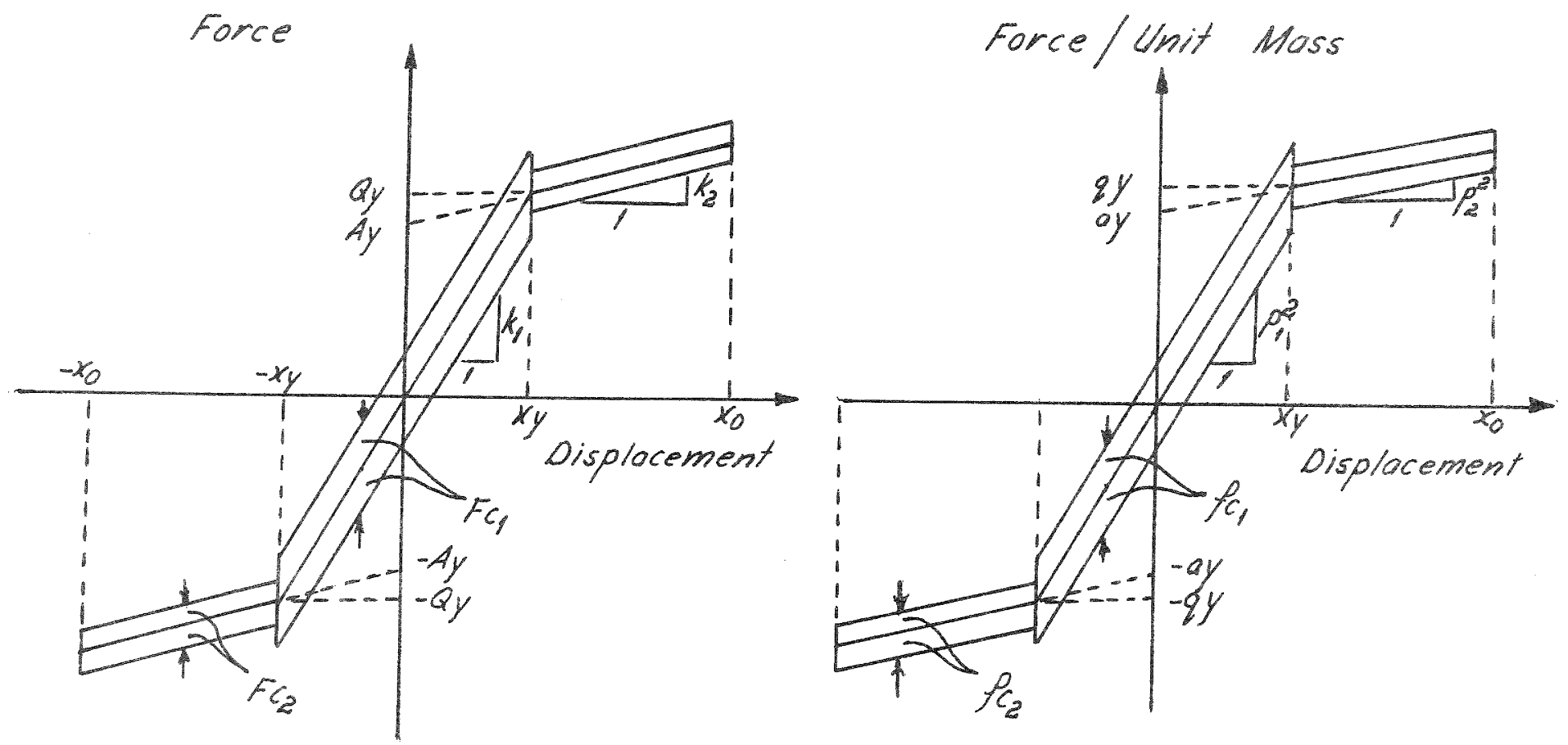

Fig. 4.

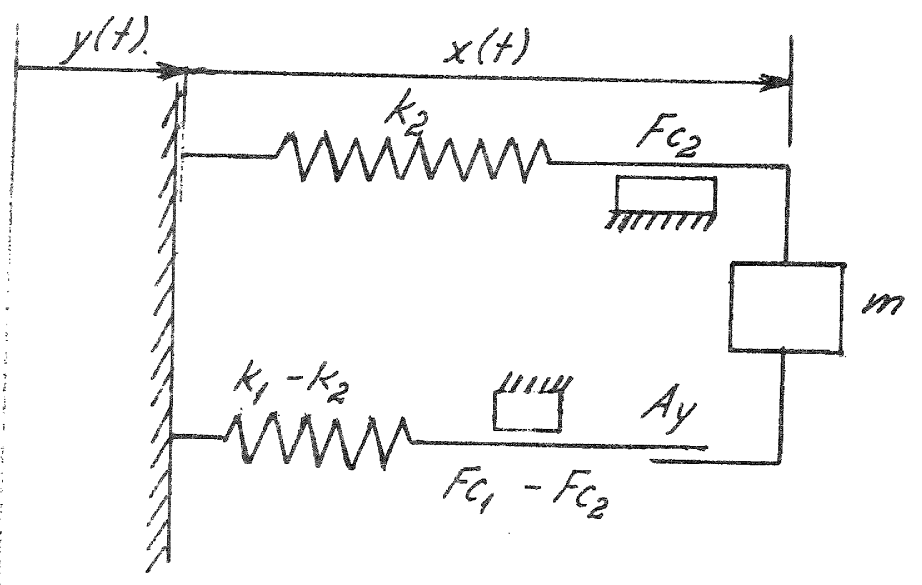

Fig. 5

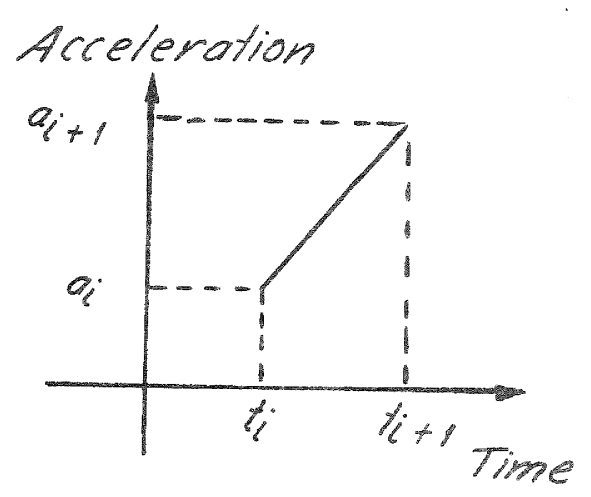

Fig. 6 

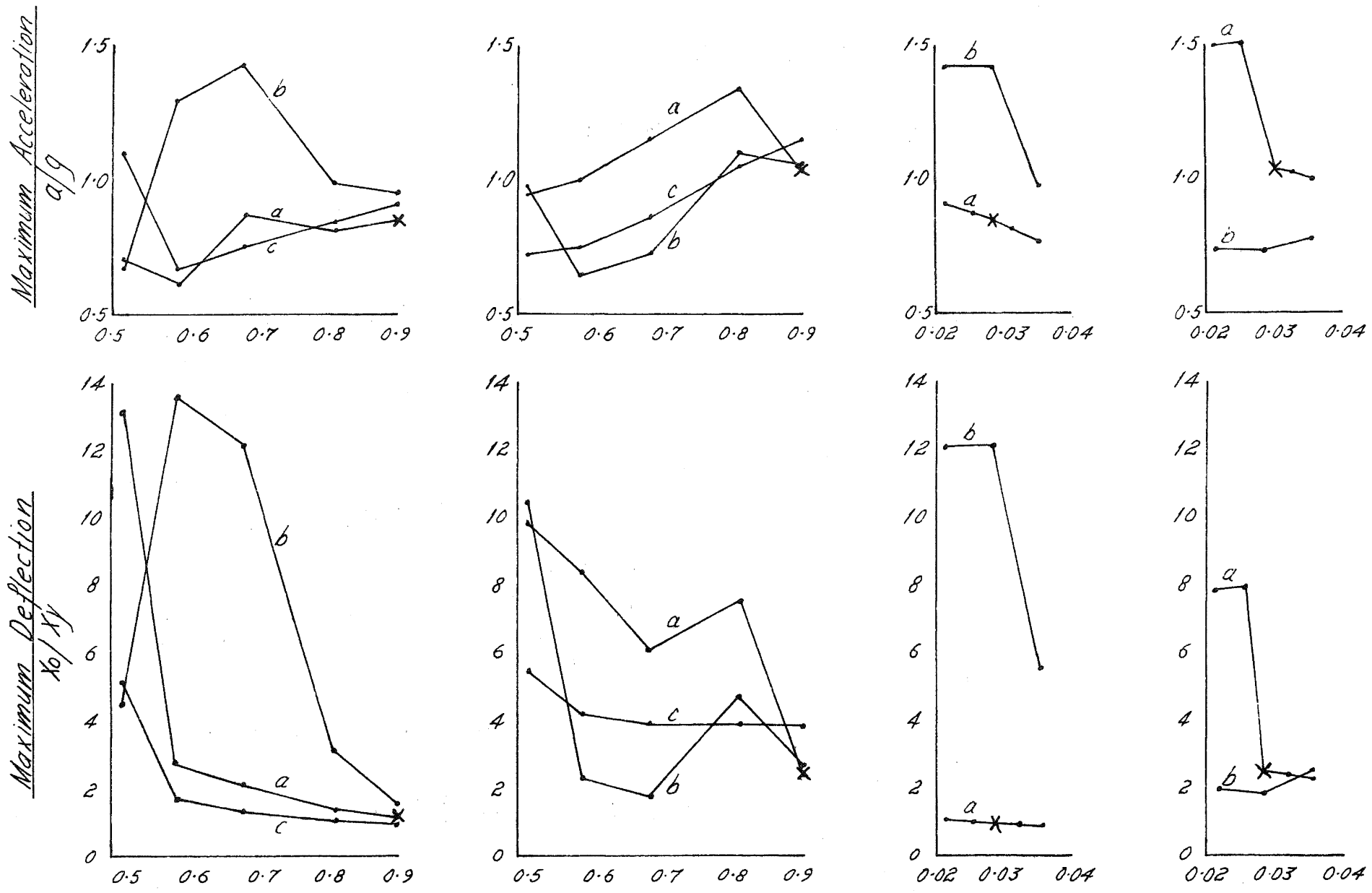

BI Earthquake.

Yeild Acceleration (ay/mg)

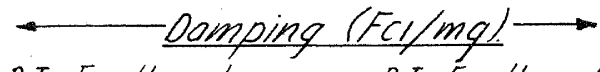

BI Earthquake.

Fig 9

Fig 10

Fig 7.

Fig 8

Earthquake Response of $220 \mathrm{kV}$ Circuit Breaker with Prestressed Porcelain Columns and Coulomb Damping - Effect of Varying Yield Level and

Note:Damping

Figs 7 and 8 - Curves a - Primary Period $=.143 \operatorname{secs}$

- " b- " "

- "c - " " = $" 286 \mathrm{secs}$

- All Curves - Coulomb Damping fc1 $=.0283 g$

Figs 9 and 10 - Curves a - Period $=.143 \mathrm{sec}$ Yjeld Level $=.9 \mathrm{~g}$

All Figures $-k_{2} / k_{1}=.1 \quad F c 2=0$

- $X=$ Response for Actual Circuit Breaker

- = " "Similar Circuit Breaker but with different Parameters. 